\title{
Spatiotemporal Dynamics of Coupled Ikeda Maps Over a Ring Network
}

\author{
Santinath Pal ${ }^{1}$ and Swarup Poria ${ }^{2}$ \\ ${ }^{1}$ Department of Mathematics, Tantigeria High School, \\ Paschim Medinipur, West Bengal, India. \\ Email: mathsanti@gmail.com \\ ${ }^{2}$ Department of Applied Mathematics, University of Calcutta, \\ Kolkata, India. \\ Email: swarup_p@yahoo.com
}

\begin{abstract}
In this paper, we investigate the spatiotemporal dynamics of a lattice of coupled chaotic Ikeda maps whose coupling connections are dynamically rewired to random sites with probability $p$. Ikeda map is defined as

$$
\begin{aligned}
& x_{n+1}=1+\left(b\left(x_{n} \cos \left(t_{n}\right)-y_{n} \sin \left(t_{n}\right)\right)\right. \\
& y_{n+1}=b\left(x_{n} \sin \left(t_{n}\right)+y_{n} \cos \left(t_{n}\right)\right)
\end{aligned}
$$

where $b$ is a positive constant and $t_{n}=0.4-6 /\left(1+x_{n}^{2}+y_{n}^{2}\right)$. Firsly, we consider a diffusively coupled network of Ikeda maps whose $x$-component can only diffuse. Bifurcation analysis of the lattice with respect to coupling strength is done. The variation of synchronized basin size with respect to coupling strength are shown for different values of rewiring probability. The variation of synchronized basin size with respect to rewiring probability are shown for different values of coupling strength. We do not observe complete synchronization in this type of network. In search for a network where complete synchronization can occur we consider a completely random network where both $x$ and $y$ components can diffuse. For the second type of network we observe synchronized spatiotemporal fixed point.
\end{abstract}

Keywords: Coupled map lattice, Networks, Spatiotemporal dynamics, Ikeda maps, Synchronization. 


\section{Introduction}

In recent years, complex networks have provided an increasingly challenging framework for the study of collective behaviors in complex systems, based on the interplay between the wiring architecture and the dynamical properties of the coupled units [1]. In recent years it has become evident that modelling large interactive systems by finite dimensional lattices on one hand, and fully random networks on the other, is inadequate, as various networks, ranging from collaborations of scientists to metabolic networks, do not fit in either paradigm [2]. In fact, many systems of biological, technological and physical significance are better described by randomizing some fraction of the regular links $[3,4,5]$. The coupled map lattice (CML) is a model which can capture the essential features of the nonlinear dynamics of extented systems [6]. A very well-studied coupling form in CMLs is the nearest-neighbour coupling. However, some degree of randomness in spatial coupling can be closer to physical reality than strict nearest neighbour scenarious. In this paper, we will study the spatiotemporal dynamics of coupled map latices(CML) of Ikeda maps where some of its coupling connections rewired randomly. Firsly, we consider a network of Ikeda maps whose $x$-component can only diffuse. The variation of synchronized basin size with respect to coupling strength are shown for different values of rewiring probability. The variation of synchronized basin size with respect to rewiring probability are shown for different values of coupling strength. Complete synchronization is not observed in this type of network. Secondly, we consider a completely random network where both $x$ and $y$ components of Ikeda maps can diffuse. The variation of basin of attraction of the synchronized state with respect to diffusion coefficient of $x$ variable is shown for fixed values of diffusion coefficient of $y$.

\section{Model}

We consider a one dimensional ring of coupled two dimensional Ikeda maps. The sites are denoted by integers $i=1,2, \ldots, N$, where $N$ is the linear size of the lattice. On each site a continuous state variable denoted by $\left(x_{n}(i), y_{n}(i)\right)$ is defined which corresponds to the physical variables of interest. The evolution of this lattice, under standard nearest neighbour interactions, in discrete time $\mathrm{n}$ is given by

$$
\begin{aligned}
& x_{n+1}(i)=(1-\epsilon) f\left(x_{n}(i), y_{n}(i)\right)+\frac{\epsilon}{2}\left\{x_{n}(i+1)+x_{n}(i-1)\right\} \\
& y_{n+1}(i)=g\left(x_{n}(i), y_{n}(i)\right)
\end{aligned}
$$

Here $\epsilon$ denotes the coupling strength. Now we will consider the above system with its coupling connections rewired randomly in varying degrees, and 
try to determine what dynamical properties are significantly affected under this rewiring. In our study, at every update we will connect a fraction $\mathrm{p}$ of randomly chosen sites in the lattice, to two other random sites, instead of their nearest neighbors as in equation (1). That is, we will replace a fraction $p$ of nearest neighbor links by random connections. The dynamical equations of this randomly rewired systems where only $x$ component of the maps can diffuse with $p$ probability are the following

$$
\begin{aligned}
& x_{n+1}(i)=(1-\epsilon) f\left(x_{n}(i), y_{n}(i)\right)+\frac{\epsilon}{2}\left\{x_{n}(\xi)+x_{n}(\eta)\right\} \\
& y_{n+1}(i)=g\left(x_{n}(i), y_{n}(i)\right)
\end{aligned}
$$

where $\xi$ and $\eta$ are random integers drawn from a uniform distribution of the integers $i=1,2, \ldots, N$, and with $(1-p)$ probability the evolution equations are

$$
\begin{aligned}
x_{n+1}(i) & =(1-\epsilon) f\left(x_{n}(i), y_{n}(i)\right)+\frac{\epsilon}{2}\left\{x_{n}(i+1)+x_{n}(i-1)\right\} \\
y_{n+1}(i) & =g\left(x_{n}(i), y_{n}(i)\right)
\end{aligned}
$$

The case $p=0$ correspond to the usual nearest neighbor interaction, while $p=1$ corresponds to completely random coupling. This scenario is like small world networks at low $p$.

Secondly, we consider completely random network of Ikeda maps of the following type. The dynamical equations of this randomly rewired network where both $x$ and $y$ components of the maps can diffuse are

$$
\begin{aligned}
& x_{n+1}(i)=\left(1-\epsilon_{1}\right) f\left(x_{n}(i), y_{n}(i)\right)+\frac{\epsilon_{1}}{2}\left\{x_{n}(\xi)+x_{n}(\eta)\right\} \\
& y_{n+1}(i)=\left(1-\epsilon_{2}\right) g\left(x_{n}(i), y_{n}(i)\right)+\frac{\epsilon_{2}}{2}\left\{y_{n}(\xi)+y_{n}(\eta)\right\}
\end{aligned}
$$

where $\xi$ and $\eta$ are random integers drawn from a uniform distribution of the integers $i=1,2, \ldots, N$.

\section{$3 \quad$ Results and Discussions}

We will now present evidence of significant effects of random rewiring on spatiotemporal order. The numerical results have been obtained by sampling a large set (100) of random initial conditions and with lattice size 100. Figure 1 (a) and 1(b) displays the bifurcation diagram of single Ikeda map with respect to bifurcation parameter $b$ for different $u$. Figure 2 shows the variation of synchronized basin size with respect to coupling strength $\epsilon$ for different rewiring probability for a network of coupled Ikeda maps. Figure 2(a) shows that basin 
(a)

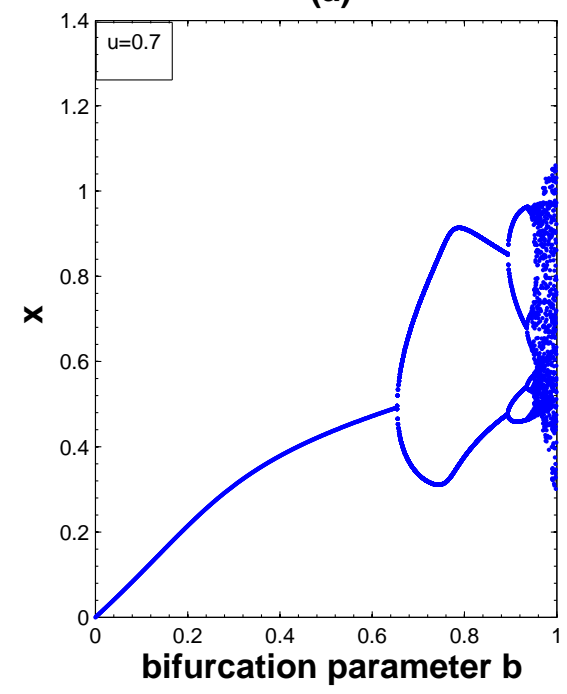

(b)

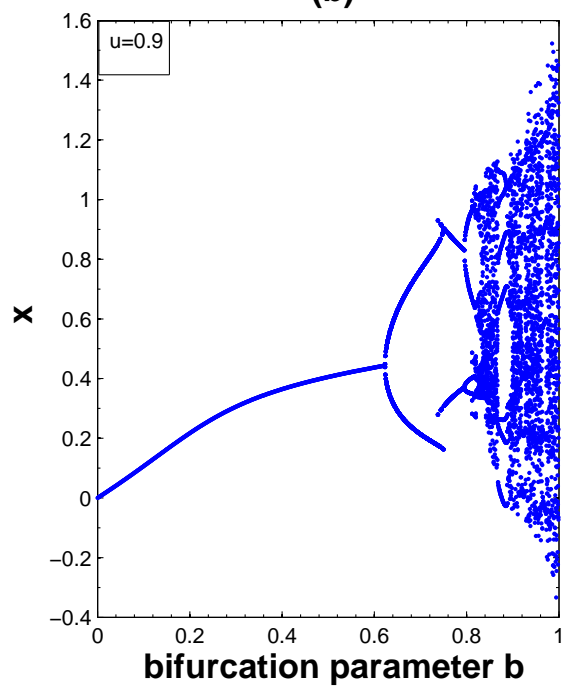

Figure 1: Bifurcation diagram of the Ikeda map with respect to bifurcation parameter $b$ (a) for $u=0.7$ and (b) for $u=0.9$.

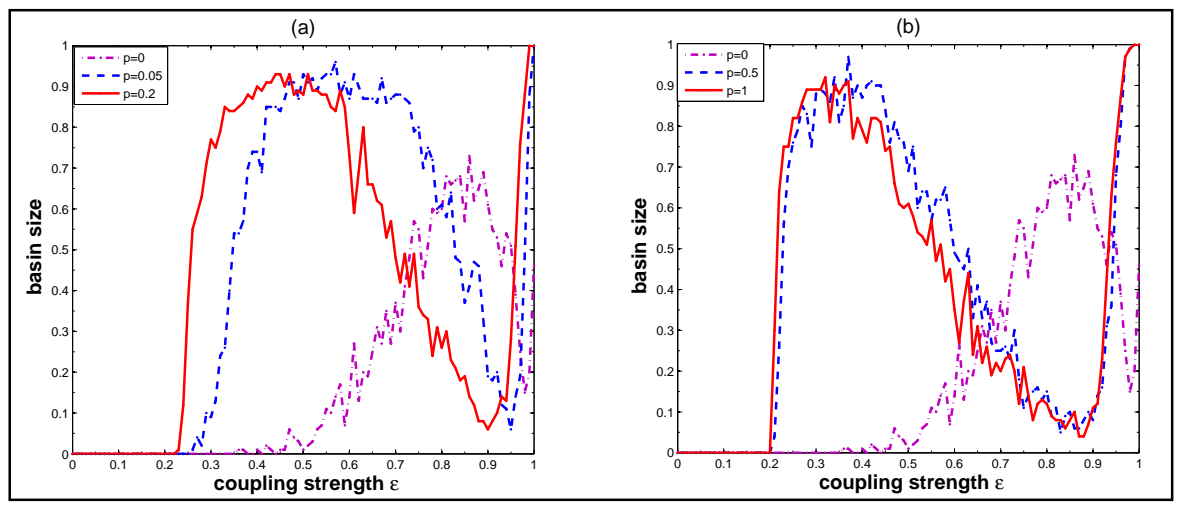

Figure 2: Size of the basin of attraction of the synchronized state with respect to coupling strength $\epsilon$ for rewiring probability (a) $p=0.0, p=0.05, p=0.2$ and (b) $p=0.0, p=0.5, p=1.0$. 

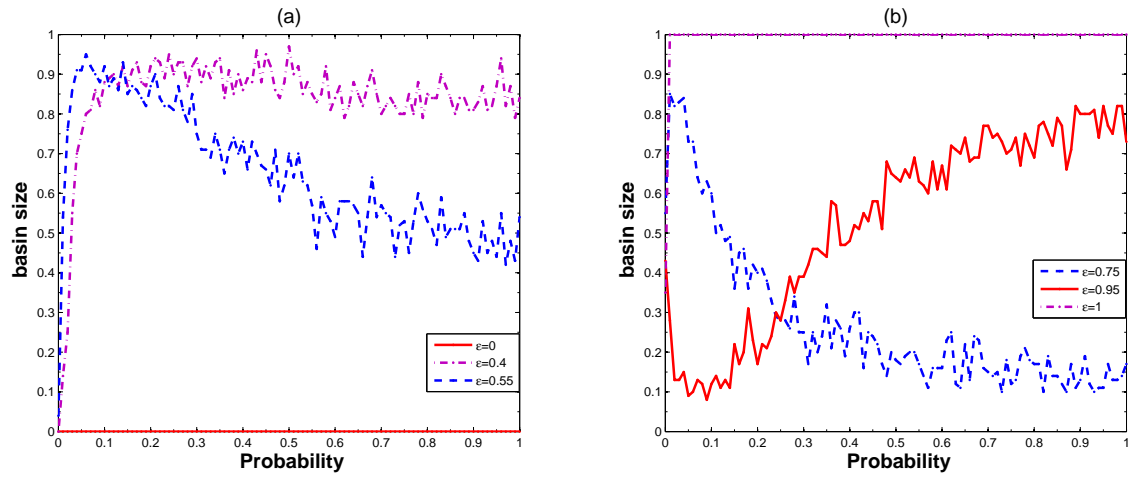

Figure 3: Size of the basin of attraction of the synchronized state with respect to rewiring probability for coupling strength $\epsilon(\mathrm{a}) \epsilon=0.0, \epsilon=0.4, \epsilon=0.55$ and (b) $\epsilon=0.75, \epsilon=0.95, \epsilon=1.0$.
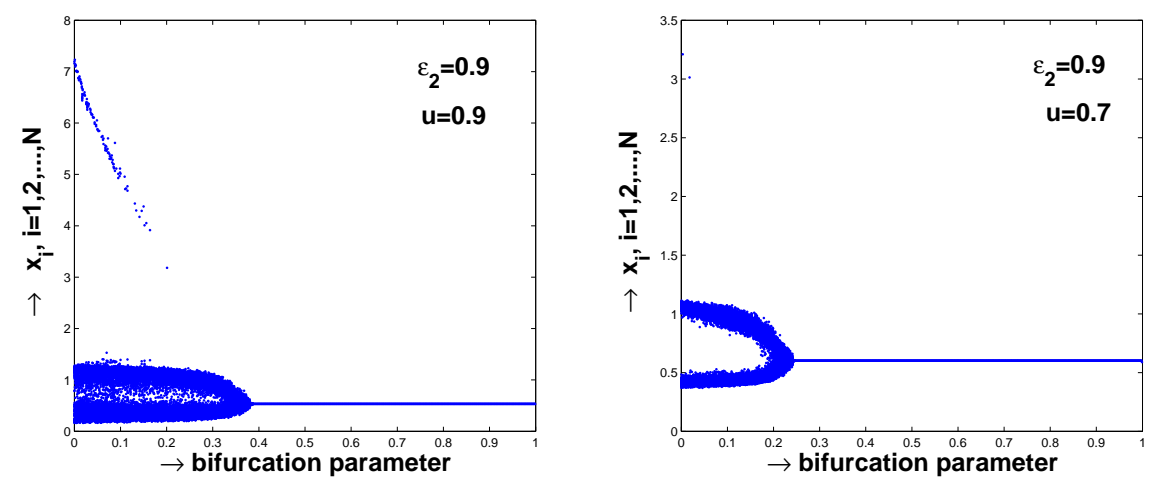

Figure 4: Bifurcation diagram with respect to coupling strength, for (a) $\epsilon_{2}=$ $0.90, u=0.9$ and for (b) $\epsilon_{2}=0.90, u=0.7$. 

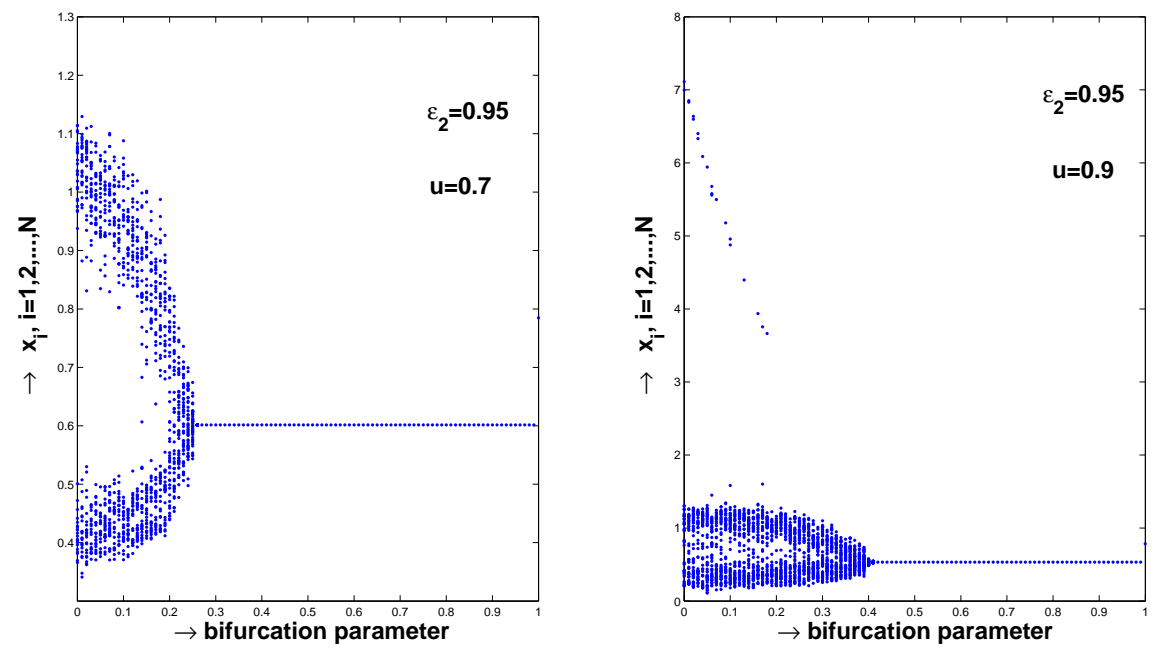

Figure 5: Bifurcation diagram with respect to coupling strength, for (a) $\epsilon_{2}=$ $0.95, u=0.7$ and for (b) $\epsilon_{2}=0.95, u=0.9$.

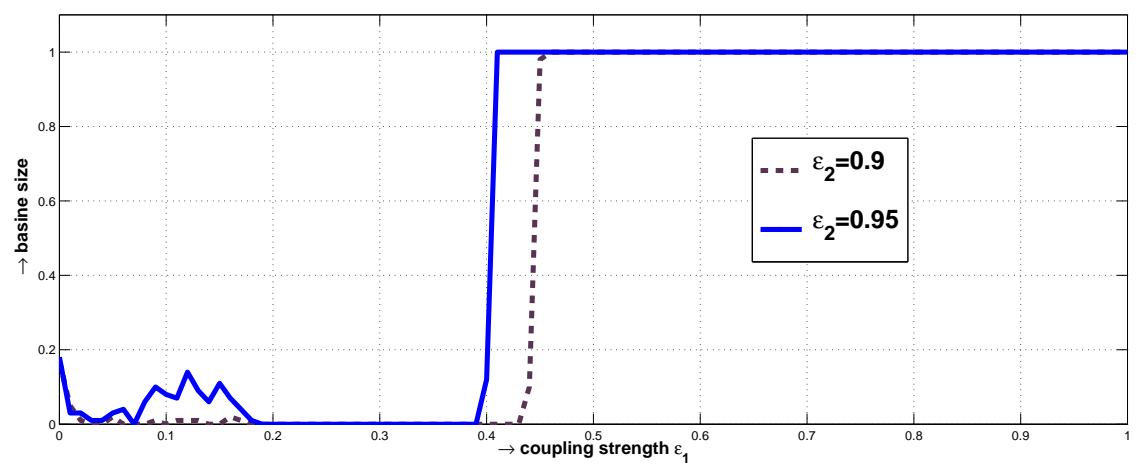

Figure 6: Size of the basin of attraction of the synchronized state with respect to rewiring probability for coupling strength $\epsilon_{2}=0.90, \epsilon_{2}=0.95, u=0.9$. 


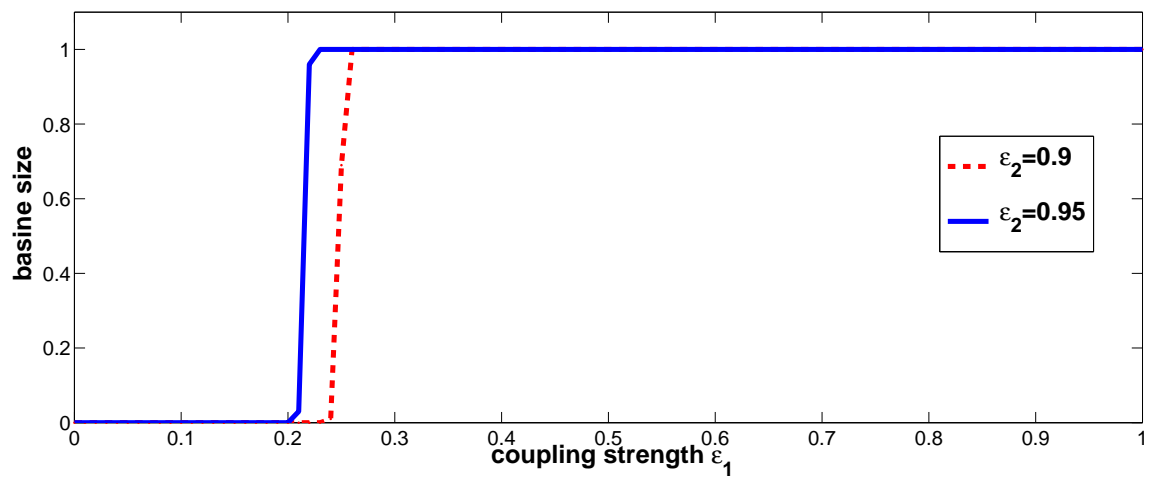

Figure 7: Size of the basin of attraction of the synchronized state with respect to rewiring probability for coupling strength $\epsilon_{2}=0.90, \epsilon_{2}=0.95, u=0.7$.

size is smallest for $p=0$, and for $p=0.05$ basin size is maximum for coupling strength $\epsilon$ in the interval $0.4 \leq \epsilon \leq 0.7$. A significant change is observed in the basin size for small non zero $p$ values compair to zero $p$ value here. In figure 3 the variation of basin size with respect to rewiring probability $p$ is shown for different coupling strength. The basin size is maximum for intermediate values of coupling strength $\epsilon$. Figure 3(a) shows the sensitivity of synchronized basin size with respect to rewiring probability $p$ for different coupling strength. From figure 3 we observe that basin size of synchronization is minimum for nearest neighbour couplinng $(p=0.0)$. The basin size increases for increasing randomness in connectivity for $0.0 \leq p \leq 0.2$. In the interval $0.2 \leq p \leq 0.5$ for increasing $p$ spatiotemporal regularity occurs for small value of coupling strength $\epsilon$. Basin size remain almost same for $0.5 \leq p \leq 0.95$. The important point is that complete synchronization is not observed here. Now we compute the bifurcation diagram of the system of equations (3) with respect to coupling strength in the figure 4 and figure 5 for different $u$ in a completely random network. We observe no synchronization for weak coupling but complete synchronization for strong coupling strength. We have calculated the synchronized basin size with respect to $\epsilon_{1}$ for different fixed $\epsilon_{2}$ in figure 6 and figure 7 . From the figure 6 we observe that complete synchronization is occuring for coupling strength $\epsilon_{1}>0.4$. for $\epsilon_{2}=0.95$ and for coupling strength $\epsilon_{1}>0.44$. for $\epsilon_{2}=0.9$ for $u=0.9$. From the figure 7 we observe that complete synchronization is occuring for coupling strength $\epsilon_{1}>0.2$. for $\epsilon_{2}=0.95$ and for coupling strength $\epsilon_{1}>0.24$. for $\epsilon_{2}=0.9$ for $u=0.7$. Therefore synchronized basin size for $\epsilon_{2}=0.95$ is greater than the basin size for $\epsilon_{2}=0.9$ in both the cases. This type of network may be useful for controlling spatially extended systems.

\section{ACKNOWLEDGEMENTS}


We are grateful to the Editor for careful reading and constructive comments.

\section{References}

[1] S. Boccaletti, V. Latora, Y. Moreno, M. Chavez and D.U. Hwang, Complex Networks: Structure and dynamics, Phys. Reports 424 (2006) 175308.

[2] S. Poria, M.D. Srimali, S. Sinha, Enhancement of spatiotemporal regularity in an optimal window of random coupling, Phys. Rev. E78(2008) 035201R.

[3] G.D. Ruxton, Low levels of immigration between chaotic populations can reduce system extinctions by inducing asynchronous regular cycles, proc. R. Soc. Lond. B 256 (1994) 189-193.

[4] K. Kaneko, Theory and applications of coupled map lattices, Jhon Wiley and Sons, New York,1993.

[5] R. Albert and A. L. Barabasi, Statistical mechanics of complex networks, Rev. Mod. Phys. 74 (2002) 47-97.

[6] D.J. Watts and S.H. Strogatz, Collective dynamics of small-world networks, Nature (London) 393 (1998) 440-442. 